\title{
KRONECKER AND HIS ARITHMETICAL THEORY OF THE ALGEBRAIC EQUATION.
}

LEOPOLD KRONECKER, one of the most illustrious of contemporary mathematicians, died at Berlin on the 29th of last December in his 68 th year.

For many years he had been one of the famous mathematicians of Germany and at the time of his death was senior active professor of the mathematical faculty of the University of Berlin and editor in chief of the Journal fiur reine und angewandte Mathematik (Crelle).

He was the last of the great triumvirate-Kummer, Weierstrass, Kronecker - to be lost to the university. Kummer retired nearly ten years ago because of sickness and old age, and recently Weierstrass followed him; but younger than the other two, Kronecker was overtaken by death in the midst of the work to which his life had been devoted. Despite his years he was much too early lost to science. The genius which had enriched mathematical literature with so many profound and beantiful researches showed no signs of weakness or weariness.

Kronecker was born at Liegnitz near Breslau in 1823. While yet a boy at the Gymnasium of his native town his fine mathematical talents attracted the notice of his master, Kummer, whose distinguished career was then just beginning. Kummer's persuasions rescued him from the business career for which he was preparing and brought him to the university.*

He studied at Breslau, whither in the meantime Kummer had been called, Bonn, and Berlin, making his degree at Berlin in 1845 with a dissertation of great value: De unitatibus complexis.

Of his instructors besides Kummer he was most influenced by Dirichlet, owing in part to Dirichlet's commanding abilities, in part to the strong arithmetical bent of Kronecker himself. As long as Dirichlet lived Kronecker's relations with him, as with Kummer, were those of the closest personal intimacy.

From the university Kronecker returned for a number of years to business and the management of his estates. But

* Kronecker makes this graceful acknowledgment of his debt to Kummer in the dedication of the Festschrift with which he honored Kummer's Doctor Jubeläum (Grundzüge einer arithmetischen Theorie der algebraischen Grössen): "In Wahrheit verdanke ich Dir mein mathematisches Dasein; ich verdanke Dir in der Wissenschaft die Du mich frủh zugewendet wie in der Freundschaft die Du mir früh entgegengebracht hast, einen wesentlichen Theil des Glücks meines Lebens." 
his mathematical activity was continuous and his fame grew apace. In 1853 he communicated to the Berlin Academy the solution of the problem : to determine all abelian equations belonging to any assigned "domain of rationality," and in $185 \%$ the first of his famous memoirs on the complex multiplication of the elliptic functions. His letter to Hermite : sur la résolution de l'équation du 5me degré in which his solution of the equation is indicated appeared in 1858.

In 1861 he was made a member of the Berlin Academy of Sciences and in $186 \%$ corresponding member of the Paris Academy.

His election to the Berlin Academy was an event of the first importance for his subsequent career, inasmuch as it was the occasion of his resuming the academic life. As member of the Academy he had the right to lecture at the University, and of this right-following the example of such men as the brothers Grimm, Kiepert, Jacobi and Borchardt-he forthwith availed himself, beginning in the winter of 1861-62 those lectures on Algebra which have for many years been one of the chief glories of Berlin. In 1883 his relations with the University were made closer still through the appointment "Professor ordinarius" and director-with Kummer and Weierstrass - of the mathematical Seminar.

The range of Kronecker's productive activity was very great. Besides distinguished work in the theory of definite integrals, he did work of the first importance in no less than three great departments of mathematics: the theory of numbers, algebra, and elliptic functions. As an arithmetician his name is associated with the great names of Gauss, Dirichlet, and Eisenstein; as an algebraist with those of Abel and Galois.

Some idea of the scope of Kronecker's contributions to mathematical literature may be conveyed by the following incomplete list of his more important memoirs: Dissertatio de unitatibus complexis (1845); Zwei Sätze über Gleichungen mit ganzzahligen Coefficienten (1857); Ueber die algebraisch auflösbaren Gleichungen $(1853,1856)$; Sur les facteurs irréductibles de l'expression $x^{n}-1$ (1854); Ueber elliptische Functionen für welche complexe Multiplication stattfindet (185\%, 1862); Ueber complexe Einheiten (1857); Sur la résolution de l'équation du 5me degré (1858); Ueber lineare Transformationen (1858); Ueber die Theorie der algebraischen Functionen (1861); Ueber die verschiedenen Factoren der Discriminanten von Eliminationsgleichungen (1865); Ueber den Affect der Modulargleichungen (1865); Ueber bilineare Formen (1868); Ueber Systeme von Functionen mehrer VTariabeln $(1869,1878)$; Ueber die verschiedenen Sturmschen Reihen und ihre gegenseitigen Beziehungen 
(18\%3) ; Zur Theorie der Elimination einer Variabeln aus zwei algebraischen Gleichungen (1881); Zur Theorie der Abelschen Gleichungen (1882); Zur arithmetischen Theorie der algebraischen Formen (1882) ; Ueber die Bernouilli'schen Zahlen (1883) ; Ueber bilineare Formen mit vier Variabeln (1883) ; Grundzüge einer arithmetischen Theorie der algebraischen Grössen (Kummer Jubeläum 1882); Zur Theorie der elliptischen Functionen (1883-1891) ; Ueber den Zahlbegriff (Zeller Jubeläum 1887). Most of his writings were published in the Berichte der Berliner Akademie or in the Journal für reine und angewandte Mathematik.

Among the finest of Kronecker's achievements were the connections which be established among the various disciplines in which he worked : notably that between the theory of quadratic forms of negative determinant and elliptic functions, through the singular moduli which give rise to the complex multiplication of the elliptic functions, and that between the theory of numbers and algebra, by his arithmetical theory of the algebraic equation.

He discovered * that to each class of quadratic forms corresponds a singular modulus which allows of complex multiplication ; to the aggregate of classes of the same determinant, an algebraic equation with rational coefficients which he showed to be irreducible; and, in fine, that the theory of quadratic forms was an anticipation of the theory of elliptic functions, the two theories being so closely related that one could have derived the notions of class and order and other fundamental properties of the quadratic forms by investigation of the properties of the elliptic function.

$\mathrm{He}$ was above all things the great arithmetician and nowhere does this appear more clearly than in his algebraic writings. It is not merely that the purely arithmetical problems growing out of algebra were attractive to him -he " arithmetized" algebra itself. In the Zeller Festschrift, after declaring his allegiance in the words of Gauss: "Die Mathematik sei die Königin der Wissenschaften und die Arithmetik die Königin der Mathematik," he writes "Und ich glaube auch, dass es dareinst gelingen wird den gesammten Inhalt aller dieser mathematischen Disciplinen (Algebra and Analysis) zu 'arithmetisen', d. h. einzig und allein auf den im engsten Sinne genommenen Zahlbegriff zu gründen, also die Modificationen und Erweiterungen dieses Begriffs wieder abzustreifen, welche zumeist durch die Anwendungen auf die Geometrie und Mechanik veranlasst worden sind."

Kronecker arrived at the conception of an arithmetical theory of the algebraic numbers and functions very early.

* Cf. Hermite: Note sur M. Kronecker, Comptes Rendus, Jan. 4, 1892. 
There are indications of it even in a letter to Dirichlet written in 1856. As its various salient concepts and theorems were discovered they were announced in the Berichte of the Academy or developed in his lectures. But he did not arrange the whole into a consecutive and complete body of doctrine until 1882 in his Grundzüge einer arithmetischen Theorie der algebraischen Grössen. Within the limits of this brief sketch it would be impossible to convey any adequate notion of this monumental work. I can attempt only to indicate the salient points of the first and more elementary of the two parts into which it is divided.

The "domain of rationalty" $\left(R^{\prime} R^{\prime \prime}\right.$. .) of any system of quantities $R^{\prime} R^{\prime \prime}$ embraces all rational functions of the $R$ 's with integral coefficients.

These $R$ 's may be quantities of any sort whatsoever, algebraic or transcendental constants or variables. In particular all the $R$ 's may equal 1 when the domain is that of rational numbers in the ordinary sense, or all the $R$ 's may be independent variables. In either of these cases the domain is said to be bounded naturally.

An integral function of one or several variables is irreducible in the domain $\left(R^{\prime} R^{\prime \prime} ..\right)$ when it contains no factor having coefficients which belong to this domain.

Every root of an irreducible algebraic equation of the $n^{\text {th }}$ degree with coefficients which belong to the domain $\left(R^{\prime} R^{\prime \prime} \ldots\right)$ is called an algebraic function of the $n^{\text {th }}$ order of the $R$ 's, the $n$ roots of the same equation being called conjugate functions.

If a single such root $G$ be "adjoined" to the $R$ 's the domain $\left(G, R^{\prime}, R^{\prime \prime} ..\right)$ is the domain of the "genus" (Gattung) $G$, the genus itself embracing those functions of the domain which are, like $G$, functions of the $n^{\text {th }}$ order.

If $G$ and $G^{\prime}$ be algebraic functions of different genera, but such that all functions of the genus $G$ belong to the domain of $G^{\prime}$, the genus $G$ is said to be contained in the genus $G^{\prime}$; and the order of $G$ is a divisor of that of $G^{\prime}$.

More than one $G$ may of course be adjoined to the $R$ 's, but it is shown that any number of $G$ 's may be replaced by a single such function which indeed is but a linear function of the given $G$ 's, with integral coefficients. In terms of this $G$ and the $R$ 's all functions of the domain $\left(G^{\prime}, G^{\prime \prime} . .: R^{\prime}, R^{\prime \prime} ..\right)$ can be expressed rationally : or the domain $\left(G^{\prime}, G^{\prime \prime} . .: R^{\prime}, R^{\prime \prime} ..\right)$ is equivalent to a domain $\left(G, R^{\prime}, R^{\prime \prime}, \ldots\right)$. This is a theorem of fundamental importance. For from it follows that in the discussion of all algebraic questions there may be selected as " elements" $R$ ', $R$, ,". of any domain of rationality whatsoever, a number of variables or indeterminates and a single algebraic function of them.

A quantity $x$ is called an integral algebraic function of the 
$R$ 's when it satisfies an equation in which the coefficient of the highest power of $x$ is 1 and the remaining coefficients are integral functions of the $R$ 's with integral coefficients. It is a fundamental theorem of the theory that for every genus $G$ there exists a finite number of such integral functions $x^{\prime}, x^{\prime \prime}, \ldots x^{(n+m)}$ in terms of which all other integral functions of the genus can be expressed linearly; i.e. in the form

$$
\phi^{\prime} x^{\prime}+\varphi^{\prime \prime} x^{\prime \prime}+\ldots+\varphi^{(n+m)} x^{(n+m)}
$$

where the $\phi$ 's are integral functions of the $R$ 's with integral coefficients. Such a system of functions $x^{\prime}, x^{\prime \prime}, \ldots x^{(n+m)}$ is called a fundamental system of the genus. In special and important cases $m$ can equal 0 .

The square of the determinant of any set of $n$ of the functions $x^{\prime}, x^{\prime \prime}, \ldots x^{(n+m)}$ and their conjugate functions is called the discriminant of these $n$ functions. The aggregate of the discriminants of every set of $n$ of the functions $x^{\prime}, x^{\prime \prime}, \ldots$ $x^{(n+m)}$ constitutes a system of rational functions of the $R$ 's, such that whatever properties are common to them all belong also to the discriminant of every set of $n$ functions of the genus and are thus characteristic of the genus itself, forming a complex of "invariants" of the genus in a higher sense of that word.

If there exist no algebraic relations among the $R$ 's, i.e. if the domain of rationality be the natural domain, there exists always an integral function of the $R$ 's with integral coefficients which is a common divisor of all the discriminants of the fundamental system of the genus and may therefore be appropriately called the discriminant of the genus itself. If $m$ equal 0 the discriminant of the $n$ elements $x^{\prime}, x^{\prime \prime}, \ldots x^{(n)}$ is itself the discriminant of the genus.

The discriminant of the genus is a divisor of the discriminant of every equation of the genus, i.e. of every equation a root of which is a function belonging to the genus, and the greatest common divisor of the discriminants of all these equations is a divisor of the $\frac{1}{2} n(n-1)$ th power of the discriminant of the genus.

Again if the genus $G^{\prime}$ be contained in the genus $G$ its discriminant will be a factor of the discriminant of $G$.

And finally the discriminant of the genus to which a set of functions belong which are defined by a system of equations $F_{1}=0, F_{2}=0, \ldots F_{n}=0$ is identical with the discriminant of this system of equations.

The demonstration of this last theorem as well as the further development of the theory necessitates a general investigation of elimination, the principal outcome of which is that the complete "resolvent" of a system of $m$ equations in $n$ quantities $x^{\prime}, x^{\prime}, x^{\prime \prime}, \ldots x^{(n)}$ is an equation of the form 


$$
F_{1}\left(x, x^{\prime}, \ldots x^{(n-1)}\right) F_{2}\left(x, x^{\prime}, \ldots x^{(n-2)}\right) \ldots F_{n}(x)=0
$$

where $x=u_{1} x_{1}+n_{2} x_{2}+\ldots+u_{n} x_{n}$, the $u$ 's being indeterminates.

The system of equations or "partial resolvents" $F_{1}=0$, $\mathrm{F}_{2}=0, \ldots \mathrm{F}_{n}=0$ is the complete equivalent of the given system. Each partial resolvent $F_{k}=0$ represents a manifoldness of $n-k$ dimensions, so that speaking geometrically a given system of equations in $n$ quantities may define simultaneously systems of points, lines, surfaces, etc.

Furthermore every divisor of the product $F_{1}, F_{2}^{\prime} \ldots F_{n}$ set equal to 0 constitutes the entire resolvent of a certain system of $n+1$ equations. Whence the important theorem : the total content of every divisor of the resolvent of a system of equations in $n$ quantities can be represented by a system of only $n+1$ equations, and therefore also a system of any number of equations can be replaced by one of only $n+1$. Any algebraic curve of double curvature, for instance, can be represented by a system of four algebraic equations.

Another most important result of this investigation of elimination is the demonstration that the concept of the algebraic function does not require any extension when systems of equations instead of single equations are brought under discussion.

This doctrine of elimination brings out the true significance of Galois' theory of algebraic equations.

Let $c_{1}, c_{2}, \ldots c_{n}$ be quantities belonging to the domain $\left(R^{\prime}, R^{\prime \prime}, \cdots\right)$ and

$$
\text { (A) } x^{n}-c_{1} x^{n-1}+c_{2} x^{n-2}-\ldots \pm c_{n}=0
$$

an irreducible equation with the roots $\xi_{1}, \xi_{2}, \ldots \xi_{n}$.

Further let $f_{1}\left(x_{1}, x_{2} \ldots x_{n}\right), f_{2}\left(x_{1}, x_{2}, \ldots x_{n}\right), \ldots$ $f_{n}\left(x_{1}, x_{2}, \ldots x_{n}\right)$ be the elementary symmetric functions defined by the identical equation

$$
\left(x-x_{1}\right)\left(x-x_{2}\right) \cdots \cdot \frac{\left(x-x_{n}\right)}{=x^{n}-f_{1} x^{n-1}+f_{2} x^{n-2}-\ldots \pm f_{n} .}
$$

Then the $n$ quantities $\xi_{1}, \xi_{2}, \ldots \xi_{n}$ may as well be regarded as determined by the system of $n$ equations

$$
\text { (B) } f_{k}\left(x_{1}, x_{2} \ldots x_{n}\right)=c_{k} \quad(k=1,2, \ldots n)
$$

as by the single equation $(A)$.

If $F(x)=0$ be the resolvent of this system $(B)$ or an irreducible part thereof, and, as above,

$$
x=u_{1} x_{1}+u_{2} x_{2}+\ldots+u_{n} x_{n},
$$

the coefficients of $F(x)$ are integral functions of the indeter- 
minates $u$ and rational functions of the $R$ 's. And since the equations $(B)$ can be satisfied only by systems of values such as

$$
x_{1}=\xi_{r_{1}}, x_{2}=\xi_{r_{2}}, \ldots x_{n}=\xi_{r_{n}}
$$

where $r_{1} r_{2} \ldots r_{n}$ is some permutation of the numbers $12 \ldots n$, $F^{\prime}(x)$ is simply the product

$$
I I\left(x-u_{1} \xi_{r_{1}}-u_{2} \xi_{r_{2}}-\ldots-u_{n} \xi_{r_{n}}\right)
$$

extended over certain of these permutations.

If now

$$
G\left(x, f_{1}, f_{2}, \ldots f_{n}\right)=0
$$

be the "Galois equation" whose $n$ ! roots are the $n$ ! functions $u_{1} x_{i_{1}}+u_{2} x_{i_{2}}+\ldots+u_{n} x_{i_{n}}$ gotten by forming the $n$ ! permutations $i_{1} i_{2} \ldots i_{n}$ of $12 \ldots n$, the coefficients of $x, f_{1} \ldots f_{n}$ in $G\left(x, f_{1}, f_{2}, \ldots f_{n}\right)$ are integral functions of the $u$ 's with integral coefficients, and one of the irreducible factors of $G\left(x, c_{1}\right.$, $c_{2}, \ldots c_{n}$ ) must be the same with $F^{\prime}(x)$. Such a factor is therefore an integral function of $x$ and the indeterminates $u_{1}, u_{2}$, ... $u_{n}$ with coefficients belonging to the domain of rationality $\left(\dot{R}^{i}, R^{\prime \prime} ..\right)$ and may be represented by $g\left(x, u_{1}, u_{2} \ldots u_{n}\right)$.

$$
g\left(x, u_{1}, u_{2}, \ldots u_{n}\right)=\Pi\left(x-u_{1} \xi_{r_{1}}-u_{2} \xi_{r_{2}}-\ldots-u_{n} \xi_{r_{n}}\right)
$$

or, if the terms of each factor be arranged with reference to the 'ं's instead of the $u$ 's

$$
g\left(x, u_{1}, u_{2}, \ldots u_{n}\right)=\Pi\left(x-u_{r_{1}} \xi_{1}-u_{r_{2}} \xi_{2}-\ldots-u_{r_{n}} \xi_{n}\right) ;
$$

that is to say $g\left(x_{1}, u_{1}, u_{2}, \ldots u_{n}\right)$, regarded as a function of the indeterminates $u$, is a function which remains unchanged for certain permutations of these $u$ 's, those represented by $r_{1}$, $r_{2}, \ldots r_{n}$.

In this manner, starting with any special equation $(A)$ one is led to general functions of indeterminates which are characteristic of the equation and have the property of maintaining their values unchanged for certain permutations of these indeterminates.

The true significance of Galois' principle thus lies in the fact that it takes as basis for the investigation of an equation the system of equations which define its conjugate roots simultaneously.

The functions $g$ to which it leads may themselves be made the starting point of the discussion. The problem then is when one replaces the indeterminates $u_{1}, u_{2}, \ldots u_{n}$ by $x_{1}$, $x_{2}, \ldots x_{n}$ : the investigation of integral functions of $n$ indeterminates $x_{1}, x_{2}, \ldots x_{n}$ with respect to the changes which they experience when the $x$ 's are permuted in all possible 
ways, the investigation taking its place in the general arithmetical theory when one regards the $x$ 's as algebraic functions of the $n$ elementary symmetric functions $f_{r}$.

If the $f$ 's take the place of the $R$ 's so that the domain of rationality is $\left(f_{1}, f_{2}, \ldots f_{n}\right)$ every rational function of the $x$ 's is an algebraic function of this domain and as such belongs to a definite genus, called simply genus of functions of $x_{1}, x_{2}, \ldots x_{n}$.

The order of the genus of any single one of the $x$ 's is $n$, that of $u_{1} x_{1}+u_{2} x_{2}+\ldots .+u_{n} x_{n}$, the "Galois genus," $n$ ! This genus contains all others and therefore their orders are all divisors of $n !$ If $\rho$ be the order of a genus $g$ and $\frac{n !}{\rho}$ be $r$, $r$ is the "number of permutations of the genus $g$," $i . e$. the number of permutations of the $x$ 's for which any function of the genus $g$ remains unchanged.

A genus $g$ is said to be a genus "proper" if after it is adjoined to the domain of rationality the equation

$$
x^{n}-f_{1} x^{n-1}+\ldots . \pm f_{n}=0
$$

remains irreducible. When such a genus $g$ is adjoined, so that the domain of rationality becomes $\left(f_{1}, f_{2}, \ldots f_{n}, g\right)$, the algebraic character of a function defined by $x^{n}-f_{1} x^{n-1}+$ . $. \pm f_{n}=0$ is changed, it falls into a special "class" of algebraic functions. All algebraic equations belong to the same class which go over into each other by rational transformation and for which the functions of the roots belonging to a definite genus $g$ belong also to the given domain of rationality $\left(R^{\prime}, R^{\prime \prime} \ldots\right)$

This characteristic property of the class of an algebraic equation and the function which it defines may be called its affect. An irreducible equation

$$
x^{n}-c_{1} x^{n-1}+\ldots \pm c_{n}=0
$$

whose coefficients belong to the domain $\left(R^{\prime}, R^{\prime \prime}, \ldots\right)$ is said therefore to have a special affect where there exists a special function of its roots, which may be called the affect-genus, which likewise belongs to the given domain. The group of permutations of this genus is called the Galois group of the equation.

The affect-genus being $g\left(x_{1}, x_{2}, \ldots x_{n}\right)$, it is the system of $n+1$ equations

$$
g=c_{0}, f_{k}=c_{k},(k=1,2, \ldots n)
$$

which by Galois' principle takes the place of the single given equation. This system is satisfied only by the $r$ systems of values

$$
x_{1}=\xi_{r_{1}}, x_{2}=\xi_{r_{2}}, \ldots x_{n}=\xi_{r_{n}}
$$


which correspond to the $r$ permutations of the genus $g$. Its order is therefore $r$ and it constitutes the irreducible part of the system $f_{k}=c_{k},(k=1,2, \ldots n)$ whose order is $n$ !

The $n$ ! functions

$x_{1}^{h_{1}} x_{2}^{h_{2}} \ldots x_{n-1}^{h_{n-1}}\left(h_{k}=0,1, \ldots n-k ; k=1,2, \ldots n-1\right)$

are the elements of a "fundamental system" of the Galois genus ; but the number of elements can be reduced to $\rho$, the order of the genus, if fractional numerical coefficients be allowed.

If the discriminant of the genus $x_{k}$, i.e.

$$
\Pi\left(x_{i}-x_{k}\right)(i, k=1,2, \ldots n ; i><k),
$$

be $D$, the discriminant of the Galois genus is $D^{\frac{1}{2} n !}$. Therefore, since the discriminant of every other genus is a divisor of that of the Galois genus and $D$ is irreducible, the discriminant of every genus is a power of $D$. From this fact it follows that for any given set of values of $f_{1}, f_{2}, \ldots f_{n}$ for which $D$ does not vanish, an infinite number of special functions of each genus can be determined all of whose conjugates differ from one another, and in terms of which every other function of the same genus can be expressed rationally. Moreover this theorem leads to a remarkably simple demonstration of the "arithmetical existence" of the roots of algebraic equations.

Upon the profound researches of the second part of the Grundzüge we cannot now enter, though this contains the heart of the arithmetical theory. Here, by aid of the "Modul-systeme" and the principle of "association" the distinctively "arithmetical" properties of the integral algebraic functions are developed, their properties, namely, when considered with respect to their divisibility by other integral functions of the same genus; and the final step is taken in the "reduction" of the domain of rationality, whereby the entire theory of tho algebraic functions is reduced to a theory of the integral functions of variables and indeterminates with integral coefficients.

Thus Kronecker's theory completes that of Galois. For it carries the general theory of equations back to a theory of indeterminates, which, before Galois, it was always assumed to be in the superficial and false sense, that the coefficients, and therefore the roots, of any equation may be treated as indeterminates.

The fine quality of Kronecker's work is even more notable than its range or the importance of its results. It possesses the rigor and elegance of the theory of numbers.

Early in the Grundzüge, when defining an irreducible func- 
tion, Kronecker remarks : "Die Definition der Irreductibilität entbehrt so lange einer sicheren Grundlage als nicht eine Methode angegeben ist, mittels deren bei einer bestimmten vorgelegten Function entschieden werden kann, ob dieselbe der aufgestellten Definition gemäss irreductibel ist oder nicht,"* and proceeds therewith to supply the missing test.

This criterion, according to which no definition may be considered justified, no theorem established, until a method is supplied for determining in every given concrete case whether the definition or theorem actually applies or not, he everywhere insisted upon, scrupulously meeting its requirements in his own work and sharply criticising all failures to meet them in the works of others. A definition which did not stand this test he denominated the invention of a mere fiction, an artificial abstraction for which there should be no place in mathematics.

This is the rigor of the ancient Greek geometry-in rejecting hypothetical constructions Euclid recognized a similar criterion-and though far enough from being always realized in the modern analysis, must characterize every mathematical theory in its finite form. For until it has been attained, either the ultimate elements of the theory have not been reached or the artificial concepts with which it has aided itself in its growth have not been set aside and the theory deduced directly from these elements.

Closely related to this fine conception of mathematical rigor are the other salient traits of Kronecker's work.

It possesses that high artistic merit which consists in the perfect adaptation of means to ends. His methods are always pure, fit, direct, and the simplest which the requirements of absolute rigor will allow. Writing to Dirichlet in 1856 he says of a method which he has discovered for deducing the properties of solvable equations of prime degree that it meets all the proper requirements of simplicity and rigor, "denn die Methode verlangt keinen irgend höheren Standpunkt mathematischen Fassungsvermögens als das Problem selbst, welches dadurch erledigt wird." $\dagger$ And again for his principle of "association" he claims: "Sie gewahrt den 'einfachsten' erforderlichen und hinreichenden Apparat, um die arithmetischen Eigenschaften der allgemeinsten algebraischen grössen 'vollständig' und ' auf die einfachste Weise' darzulegen," adopting the phrases which are quoted from the first proposition of Kirchhoff's Mechanics. This "Einfachheit," to be sure, is of a kind which it oftentimes requires

* Grundzüge, etc., p. 11.

† Göttinger Nachrichten, 1885, p. 364.

$\ddagger$ Grundzüge, etc., p. 93 . 
much reflection to appreciate. He was a foe not only of artificial concepts but of all artificial methods and of all artificial or purely formal tendencies in mathematics. He would have rid mathematics of the artificial numbers and of its "symbolic" methods, and the devising of new functions seemed to him a foolish waste of energy. "God created numbers and geometry," I once heard him say, "but man the functions."

It was his boast that he was the most practical of matbematicians. He said whimsically to me one day last summer : "It is a pity that you, Americans, do not know me better. You would surely appreciate me, I am so practical." And in a somewhat transcendental sense of the word, to be sure, he was profoundly practical. He sought to avoid all mere abstractions and to give his theories concrete form. Thus in the Galois theory he replaced the abstraction, a group of substitutions, by concrete functions which remain unchanged for the substitutions of the group. Neither definition, theorem, nor method had value in his eyes which could not be applied to concrete cases, which could not be made to yield concrete results. On this account he did not set great store by the services of the theory of substitutions to algebra. With all its beauty, he would urge, it is only formal, it does not show how to construct the group of a given equation.

Kronecker influenced the mathematical thinking of Germany as much through his lectures as through his published writings. He was a very stimulating and interesting lecturer. To an unusual degree he took his hearers into his confidence and allowed them the privilege of watching the actual evolution of his thoughts. His lectures were not overprepared, but the details of even important demonstrations were left to take their chances in the lecture room. Occasionally there would be a disastrous slip in the reckoning or argument, or the outcome would be the discovery that the theorem sought to be established was false. But that only afforded opportunity to see the marvellous quickness with which he would run an error down and recover himself.

His lectures were always fresh. The principal courses were on determinants, theory of numbers, algebra, and definite integrals, and one of these in its turn he delivered each semester. But he never merely repeated himself. If a lecture did not differ from all its predecessors in content, it surely did in point of view or method. It was always the most recent product of his mathematical thinking.

In his lecturing, moreover, he avoided the excessive conciseness, which is the chief cause of the difficulty of his published writings.

Personally, Kronecker was most charming and amiable, a polished gentleman and man of the world. He was very gen- 
erous with his time and thoughts, loving to talk to an appreciative listener of some favorite doctrine, or of the famous mathematicians with whom he had been associated.

He was a man of rare genius, a mathematician of the first rank in this century of great mathematicians.

Princeton College, April 20, 1892.

Henry B. Fine.

\section{MULTIPLICATION OF SERIES.}

BY PROF. FLORIAN CAJORI.

THE salient feature of the new era which analysis entered upon during the first quarter of this century is vividly illustrated in the history of infinite series. Extending from that time back to Newton we have a formal period which gave rise to general theorems, the validity of which was not thoroughly tested. Thus, in series, there were put forth during that epoch the binomial theorem, the theorems of Taylor, Maclaurin, John Bernoulli, and Lagrange. Infinite series were used by Newton, Leibnitz, and Euler in the study of transcendental functions. As a rule, the convergency of expressions was not ascertained, and the confusion which prevailed in the theory of series gave rise to curious paradoxes. But with the advent of Gauss, Cauchy, and Abel, began the new era which combined dexterity in form with rigor of demonstration.

In the multiplication of series, mathematicians of the earlier period considered simply the form of the products and hardly ever thought of inquiring further into the validity of the operation. Reliable tests for convergency were unknown. The product of any two infinite series was accepted with nearly the same degree of confidence as was the product of finite expressions. Thus, De Moivre* extended the binomial formula to infinite series and deduced the following formula: $\left(a z+b z^{2}+\ldots\right)^{m}$

$$
=a^{m} z^{m}+\frac{m}{1} a^{m-1} b z^{m+1}+\frac{m}{1} \cdot \frac{m-1}{2} a^{m-2} b^{2} z^{m+2}+\ldots
$$

This was accepted as true without any limitations whatever.

* A method of raising an infinite multinomial to any given power, or extracting any given root of the same. Philosophical Transactions, No. 230,1697 . 